\title{
Loss of Tyrosine Kinase 2 Does Not Affect the Severity of Jak2V617F-induced Murine Myeloproliferative Neoplasm
}

\author{
TAKUMI YAMAJI, KOTARO SHIDE, TAKURO KAMEDA, MASAAKI SEKINE, AYAKO KAMIUNTEN, \\ TOMONORI HIDAKA, YOKO KUBUKI, HARUKO SHIMODA, HIROO ABE, \\ TADASHI MIIKE, HISAYOSHI IWAKIRI, YOSHIHIRO TAHARA, MITSUE SUETA, \\ SHOJIRO YAMAMOTO, SATORU HASUIKE, KENJI NAGATA and KAZUYA SHIMODA
}

Department of Gastroenterology and Hematology, Faculty of Medicine, University of Miyazaki, Miyazaki, Japan

\begin{abstract}
Background/Aim: In myeloproliferative neoplasms $(M P N)$, Janus kinase 2 (JAK2) is activated by mutations including JAK2V617F (JAK2VF). It is unclear whether JAK kinases [i.e. JAK1, JAK2, JAK3, or tyrosine kinase 2 (TYK2)] other than JAK2 have cooperative actions such as enhancement or suppression of JAK2. If other kinases enhance activation, therapies that co-target them could have a therapeutic efficacy. We examined the role of TYK2 in Jak2VF-induced murine MPN. Materials and Methods: We crossed Jak2VF transgenic mice and Tyk2-knockout (Tyk2KO) mice to generate Jak2VF/Tyk2KO mice. The disease severity and treatment effect with a JAK2 inhibitor was compared between Jak2VF and Jak2VF/Tyk2KO mice. Results: Both types of mice developed MPN, and there were no differences in peripheral blood counts, spleen weight, or survival period. Upon JAK2 inhibitor therapy, both types of mice had equally improved leukocytosis and splenomegaly. Conclusion: TYK2 does not have cooperative effects with JAK2VF upon MPN onset nor in the presence of a JAK2 inhibitor.
\end{abstract}

Classical breakpoint cluster region-Abelson $(B C R-A B L)$ negative myeloproliferative neoplasms (MPN) includes three main diseases of distinct clinical presentation, namely polycythemia vera, essential thrombocythemia, and primary myelofibrosis. The most recurrent abnormality is a gain-offunction substitution of valine to phenylalanine at position 617 (V617F) in Janus kinase 2 (JAK2) (1). In patients with MPN, clonal expansion of myeloid cells by JAK2 activation

Correspondence to: Dr. Kotaro Shide, Department of Gastroenterology and Hematology, Faculty of Medicine, University of Miyazaki, 5200 Kihara, Kiyotake, Miyazaki 889-1692, Japan. Tel: +81 985859121, Fax: +81 985855194, e-mail: koutaro_shide@ med.miyazaki-u.ac.jp

Key Words: Myeloproliferative neoplasms, JAK2, TYK2, animal models, JAK2 inhibitors. results in leukocytosis, hepatosplenomegaly associated with extramedullary hematopoiesis, and constitutional symptoms due to cytokine overproduction. JAK2 inhibitors alleviated these symptoms in clinical trials, and ruxolitinib, the first JAK2 inhibitor approved for the treatment of MPN, is now in widespread clinical use $(2,3)$. However, in ruxolitinibtreated patients, the therapeutic effects plateau in a few months, and no clear effects on the mutant allele burden have been observed, indicating the presence of resistance to JAK2 inhibitor. As a possible resistance mechanism, it was shown in vitro that JAK2 can form a heterodimer with the nonreceptor tyrosine protein kinase 2 (TYK2) or JAK1 in the presence of JAK2 inhibitor, thereby enhancing downstream signaling (4). In addition, it is unclear whether Janus kinases (i.e. JAK1, JAK2, JAK3, and TYK2) other than JAK2 have cooperative effects such as enhancement or suppression of activated JAK2. If other kinases in addition to JAK2 activate or induce drug resistance, therapies that co-target them might have therapeutic efficacy. In this study, we used a Jak2V617F (Jak2VF)-induced murine MPN model to determine whether TYK2 promoted the severity of MPN in cooperation with JAK2VF, and whether the loss of TYK2 influenced the therapeutic effects of a JAK2 inhibitor.

\section{Materials and Methods}

Mouse models. Transgenic mice that expressed the Jak2VF mutation (Jak2VF mice) and Tyk2-knockout mice (Tyk2KO mice) were established and analyzed as previously described $(5,6)$. We used $J a k 2 \mathrm{VF}$ mice that showed a spectrum of clinicopathological features of human primary myelofibrosis. Jak2 VF mice and Tyk2 KO mice were crossed to create $J a k 2 \mathrm{VF} / T y k 2 \mathrm{KO}$ mice. To investigate the influence of TYK2 loss on MPN severity induced by JAK2VF, wild-type (WT), Jak2VF, and Jak2VF/Tyk2KO mice were analyzed. Peripheral blood (PB) cell counts were assessed every 8 weeks after birth, and the survival time of these mice was also examined. Another group of mice was sacrificed at 3,6, and 9 months after birth, and their organ weights were assessed followed by histopathology. To investigate the therapeutic effects of JAK2 inhibitor, 12-week-old WT, Jak2VF, and Jak2VF/Tyk2KO mice 
were treated by oral gavage twice daily with $50 \mathrm{mg} / \mathrm{kg}$ JAK2 inhibitor or vehicle $(0.5 \%$ methylcellulose) for 16 weeks. PB cell counts were assessed every 4 weeks, and mice were sacrificed at the study endpoint and analyzed. Animal studies were performed in accordance with the Ethics Committee of the University of Miyazaki (approval number 2014-520). For pathological examination, tissue samples were fixed in formalin, embedded in paraffin blocks, and sectioned for hematoxylin and eosin (H\&E) or Gomori silver staining. Fluorescence-activated cell sorting (FACS) analysis was performed as previously described (5).

$N$-[(1S)-1-(4-Fluorophenyl)ethyl]-4-(1-methyl-1H-pyrazol-4-yl)$N 0$-(pyrazin-2-yl)pyridine-2,6-diamine maleate, a potent and selective inhibitor of JAK2 and SRC-family of protein tyrosine kinases, which is currently in phase 2 clinical trials for MPN, was kindly provided by Discovery Research Laboratories, Nippon Shinyaku Co., Ltd. (Kyoto, Japan) (7).

Quantitative real-time polymerase chain reaction ( $q P C R)$. RNA was extracted from the bone marrow (BM) cells of WT, TYK2KO, $J A K 2 \mathrm{VF}$, and $J A K 2 \mathrm{VF} / T Y K 2 \mathrm{KO}$ mice. Then the expression levels of Jak1, Jak2, Jak3, and Tyk2 were measured by qPCR as previously described (8).

Statistical analysis. Results are presented as the mean \pm standard error of the mean (SEM). For the comparison of hematological values, analysis of variance (ANOVA) with repeated measures was used. To assess statistical significance among groups, Tukey's Honest Significant Difference (HSD) test with one-way ANOVA was used. Statistical analysis of the survival was performed with the log-rank test. $p$-Values less than 0.05 were considered statistically significant.

\section{Results}

Loss of TYK2 did not affect disease severity of JAK2VFinduced murine MPN. The expression levels of Jakl, Jak2, $J a k 3$, and $T y k 2$ in the BM cells of WT, Jak2VF, and $J a k 2 \mathrm{VF} / T y k 2 \mathrm{KO}$ mice were measured by qPCR $(\mathrm{n}=3-5)$, and the results are shown in Figure 1A. Tyk2 expression was not detected in Jak2VF/Tyk2KO mice, and was 0.7 -fold lower in $J a k 2 \mathrm{VF}$ mice than in WT mice $(p<0.01)$. Due to transgene expression, Jak2 expression was 1.7-fold and 1.4-fold higher in Jak2VF and Jak2VF/Tyk2 KO mice, respectively, than in WT mice. Jak 1 expression was 0.8 -fold and 0.7 -fold lower in $\mathrm{Jak} 2 \mathrm{VF}$ and $J a k 2 \mathrm{VF} / T y k 2 \mathrm{KO}$ mice, respectively, than in WT mice. The expression of Jak 3 did not differ among the three groups. All JAK kinases, with the exception of TYK2, were equally expressed in Jak2 $\mathrm{VF}$ and $J a k 2 \mathrm{VF} / T y k 2 \mathrm{KO}$ mice.

Next, we compared the phenotype of WT, Jak2VF, and $J a k 2 \mathrm{VF} / T y k 2 \mathrm{KO}$ mice. Both Jak2VF mice and $J a k 2 \mathrm{VF} / T y k 2 \mathrm{KO}$ mice showed increased white blood cell count compared with WT mice (Figure 1B), and also showed progressive anemia. The platelet count of Jak2 $\mathrm{VF}$ and $J a k 2 \mathrm{VF} / T y k 2 \mathrm{KO}$ mice was markedly higher than that of WT mice at 8 weeks of age, but decreased thereafter. There was no difference in $\mathrm{PB}$ cell count between Jak2VF and $J a k 2 \mathrm{VF} / T y k 2 \mathrm{KO}$ mice. FACS analysis of PB cells showed that macrophage-antigen-1-positive/granulocyte-differentiation antigen-1-positive $\left(\mathrm{Mac1}^{+} / \mathrm{Gr}^{+}\right)$myeloid cells were increased and B-cell isoform of 220-kDa-positive (B220 ${ }^{+}$) B-cells were decreased in both Jak2VF and Jak2VF/Tyk2KO mice compared to WT mice, but there were no differences between $J a k 2 \mathrm{VF}$ and $J a k 2 \mathrm{VF} / T y k 2 \mathrm{KO}$ mice (data not shown).

Regarding organ weight, significant hepatomegaly and splenomegaly were observed in Jak2VF and Jak $2 \mathrm{VF} / T y k 2 \mathrm{KO}$ mice compared to WT mice $(p<0.01)$, but no difference was observed between Jak2VF and $J a k 2 \mathrm{VF} / T y k 2 \mathrm{KO}$ mice, even after 9 months of observation (Figure 1C). The survival of Jak2VF and Jak2VF/Tyk2KO mice was worse than that of WT mice $(p<0.01)$, and as with leukocytosis and organomegaly, there was no difference between these two groups of mice (Figure 1D).

Loss of TYK2 did not affect the therapeutic efficacy of a $J A K 2$ inhibitor. We determined whether the loss of TYK2 affected the therapeutic efficacy of a JAK2 inhibitor. At 12 weeks of age, all of the Jak2VF and Jak2VF/Tyk2KO mice developed MPN, exhibiting leukocytosis with an average white blood cell count of $7-8 \times 10^{10} / 1$ (Figure 2A). Jak2VF and Jak2 $\mathrm{VF} / T y k 2 \mathrm{KO}$ mice were divided into two groups: one group received an oral gavage dose of $50 \mathrm{mg} / \mathrm{kg}$ JAK2 inhibitor twice daily (Jak2VF-inh; n=16, Jak2VF/Tyk2KOinh; $\mathrm{n}=16)$ and the other group received vehicle twice daily (Jak2VF-veh; n=16, Jak2VF/Tyk2KO-veh; n=16) for 16 weeks. As a control for vehicle-treated mice, WT mice were treated with vehicle (WT-veh; $n=16$ ) were used. vehicletreated Jak2VF and Jak2VF/Tyk2KO mice showed marked leukocytosis compared with vehicle-treated WT mice. Significant suppression of leukocytosis was observed by administration of JAK2 inhibitor in both treatment groups (Jak2VF: $p<0.05$; Jak2VF/Tyk2KO: $p<0.01$ ) (Figure 2A). Inhibitor-treated Jak2 $\mathrm{VF}$ and $J a k 2 \mathrm{VF} / T y k 2 \mathrm{KO}$ mice showed no difference in leukocyte count, and there was also no difference in the therapeutic effects of JAK2 inhibitor between the two groups. In the PB cells of vehicle-treated $J a k 2 \mathrm{VF}$ and Jak2VF/Tyk2KO mice, $\mathrm{Mac}^{+} / \mathrm{Grr}^{+}$myeloid cells increased and $\mathrm{B} 220^{+} \mathrm{B}$-cells and $\mathrm{CD}^{+}$T-cells decreased compared with vehicle-treated WT mice, but the proliferation of myeloid cells was suppressed and the B- and T-lymphocyte fractions were restored by treatment with JAK2 inhibitor (Figure 2B). There were no differences in leukocyte fractions between inhibitor-treated Jak2 VF and $J a k 2 \mathrm{VF} / T y k 2 \mathrm{KO}$ mice. In the vehicle-treated groups of transgenic mice, progressive anemia was observed, but in the JAK2 inhibitor-treated groups, anemia progression stopped, although the difference was not significant (Figure 2A). No significant differences in hemoglobin levels were observed between JAK2 inhibitor-treated groups. Vehicle-treated $J a k 2 \mathrm{VF}$ and Jak2VF/Tyk2KO mice showed thrombocytosis compared to WT mice treated with vehicle in the early 


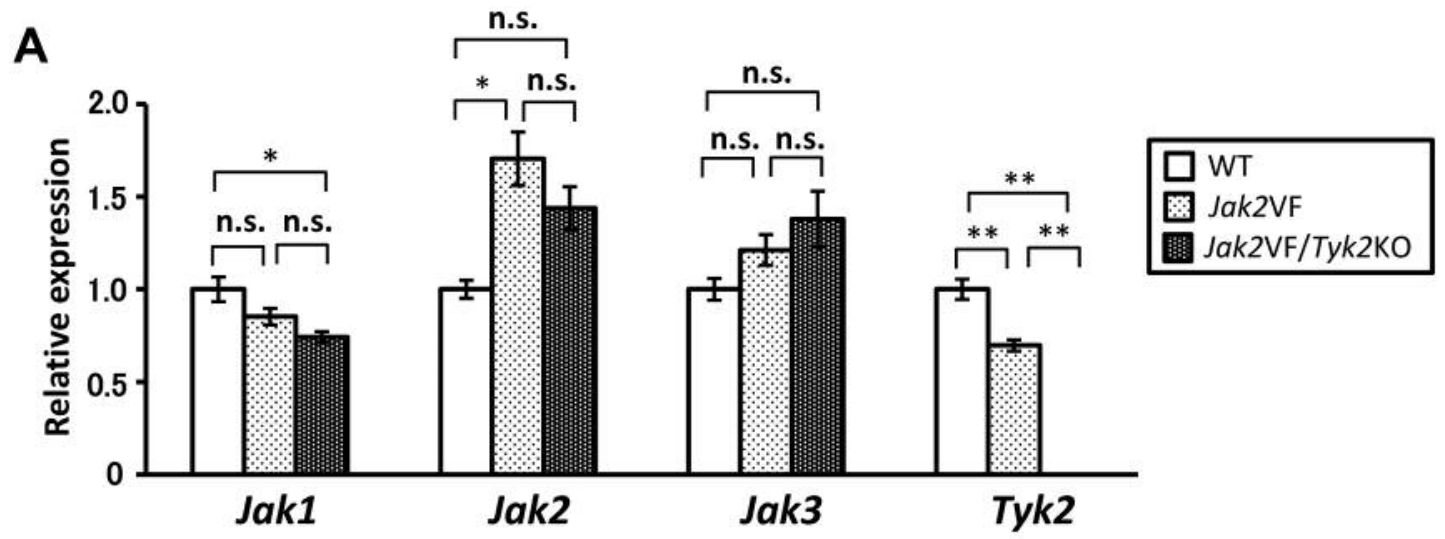

B
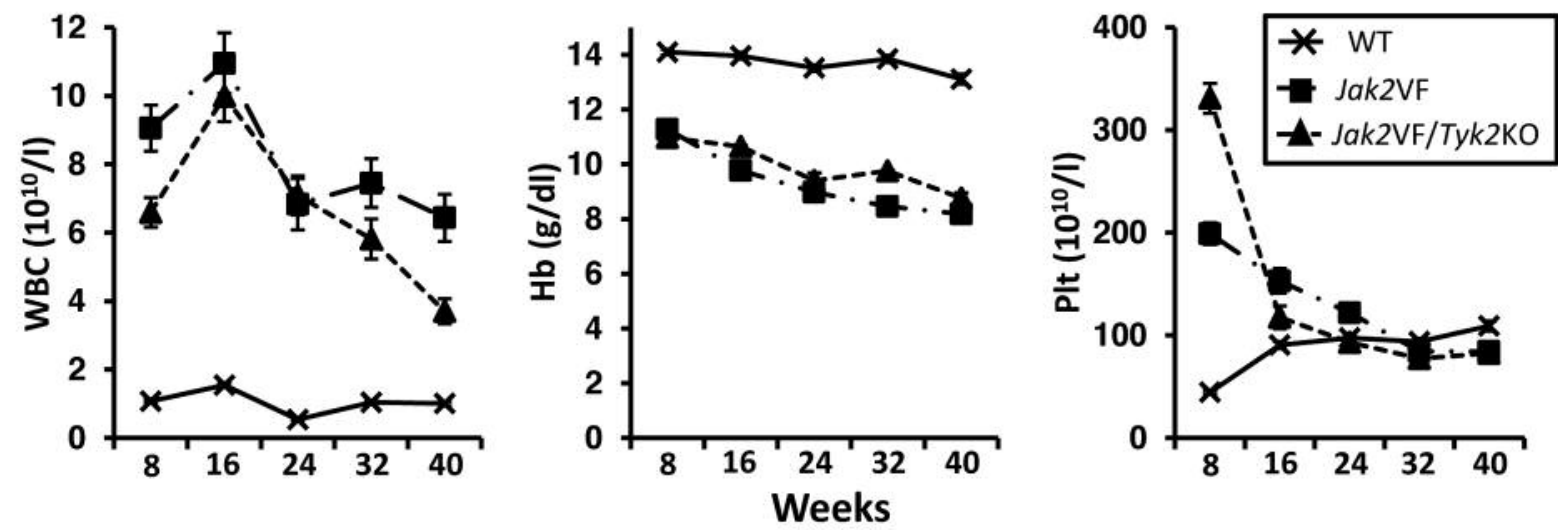

C
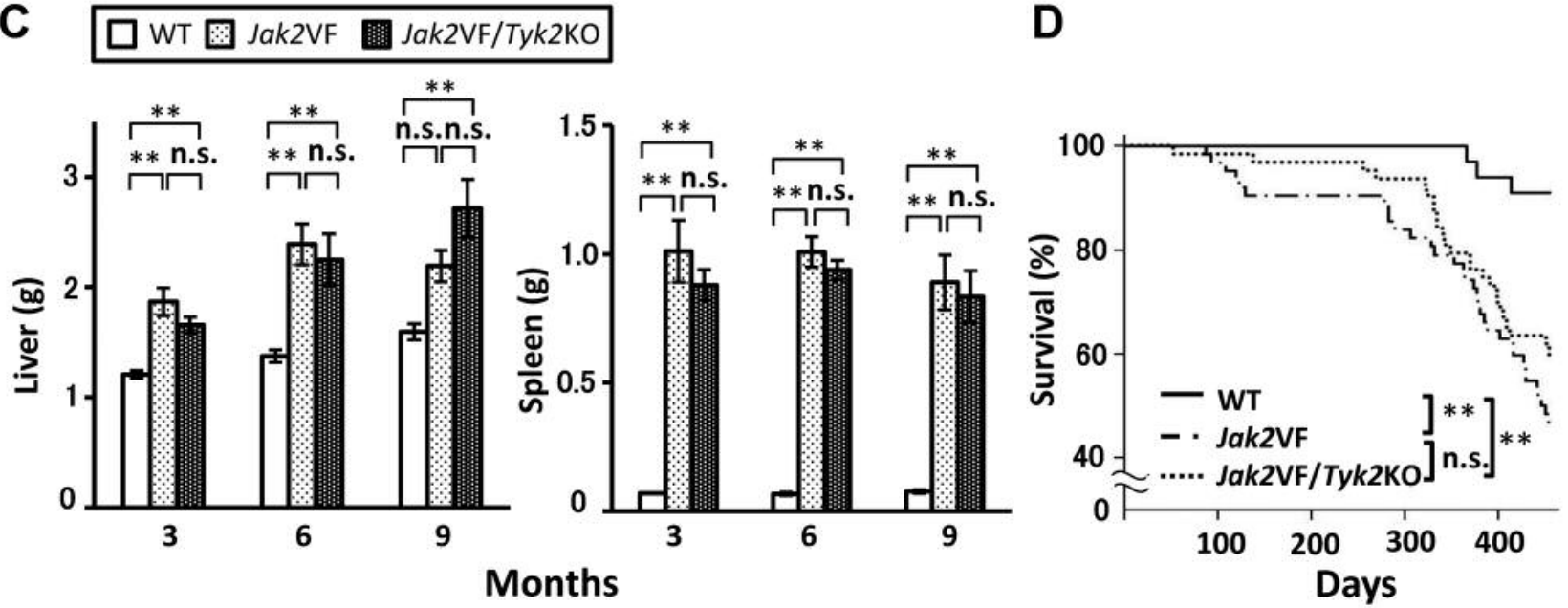

Figure 1. Tyrosine kinase 2 (TYK2) deficiency does not affect the severity of myeloproliferative neoplasms in mice. A: The mRNA expression levels of Janus kinases (JAK) in bone marrow cells from wild-type (WT) mice (n=3), JAK2 V617F transgenic (Jak2VF) mice (n=5), and Jak2VF/tyrosine kinase 2 gene knockout (Tyk2KO) mice (n=4). The expression level in WT mice was set at 1. Tukey's HSD test with one-way ANOVA was used. B: Differential blood counts in WT mice $(n=28)$, Jak2VF mice $(n=34)$, and Jak2VF/Tyk2KO mice $(n=35)$ every 8 weeks after birth. ANOVA with repeated measures was used. Significant differences for all counts vs. WT: Jak2VF, $p<0.01 ; J a k 2 V F / T y k 2 K O, p<0.01$. C: Liver and spleen weights at 3, 6, and 9 months after birth (n=5). Tukey's HSD test with one-way ANOVA was used. D: Kaplan-Meier survival curves of WT mice ( $n=33)$, Jak2VF mice (n=62), and Jak2VF/Tyk2KO mice (n=63) mice. Log-rank test was used. WBCs, White blood cells; Hb, hemoglobin; Plt, platelets. Data are presented as the mean \pm SEM. Significantly different at $* p<0.05$ and $* * p<0.01$. 

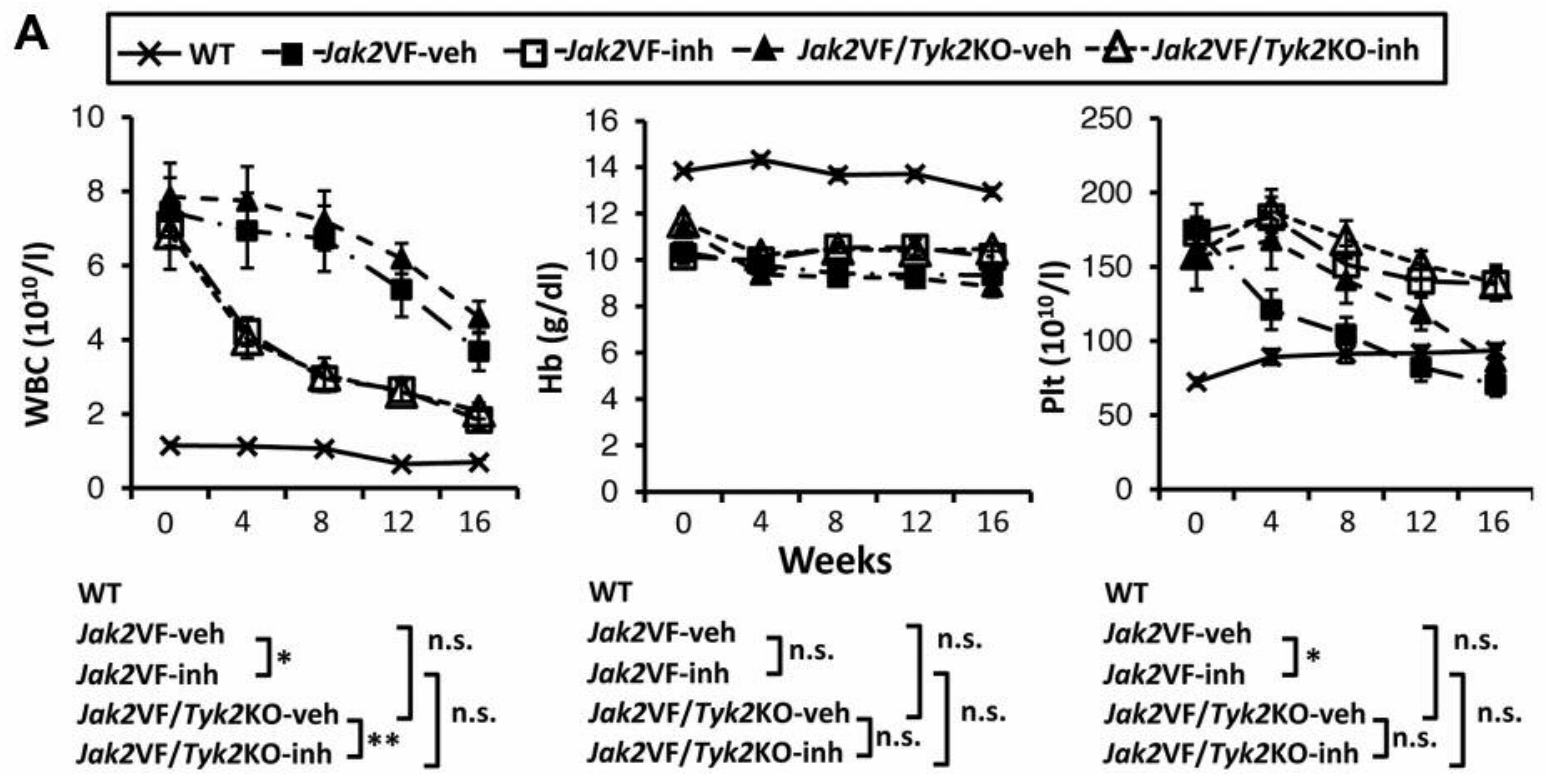

WT

WT
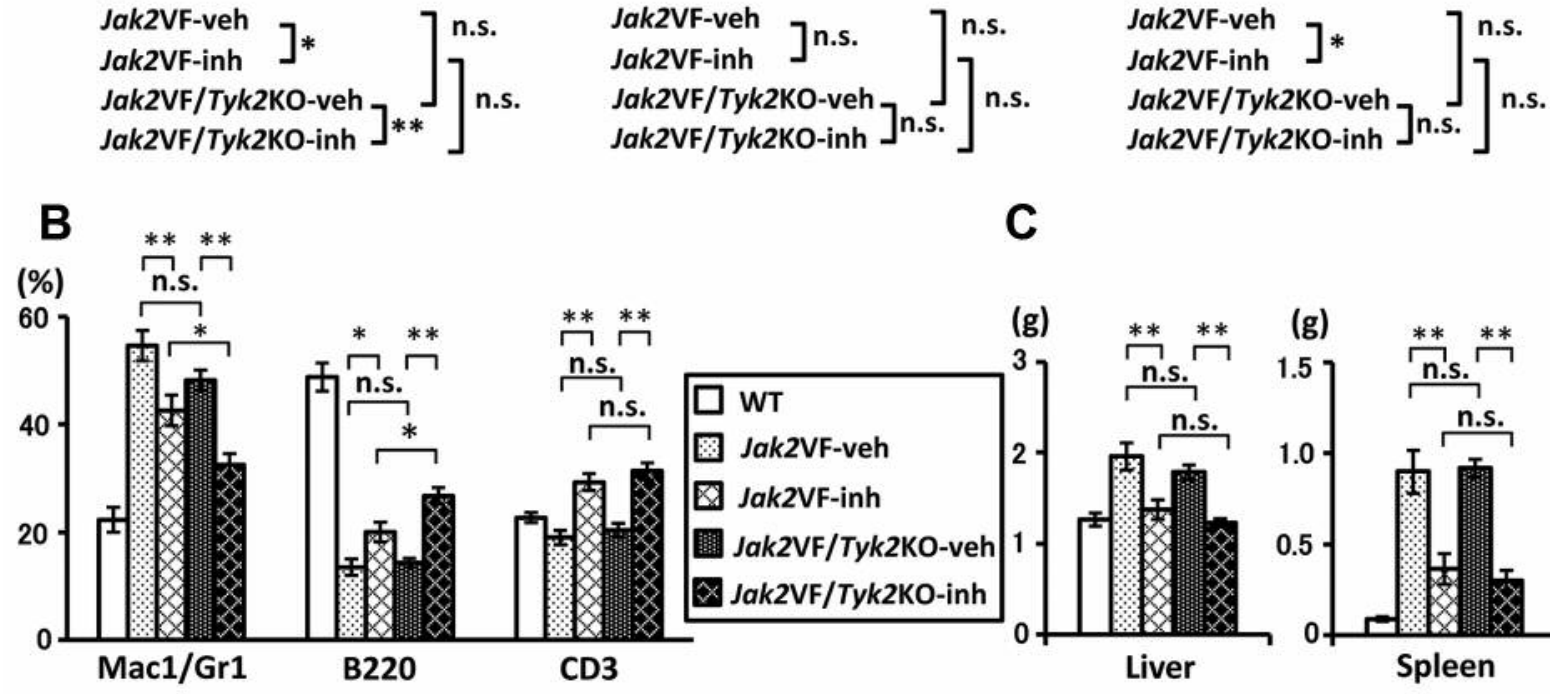

C
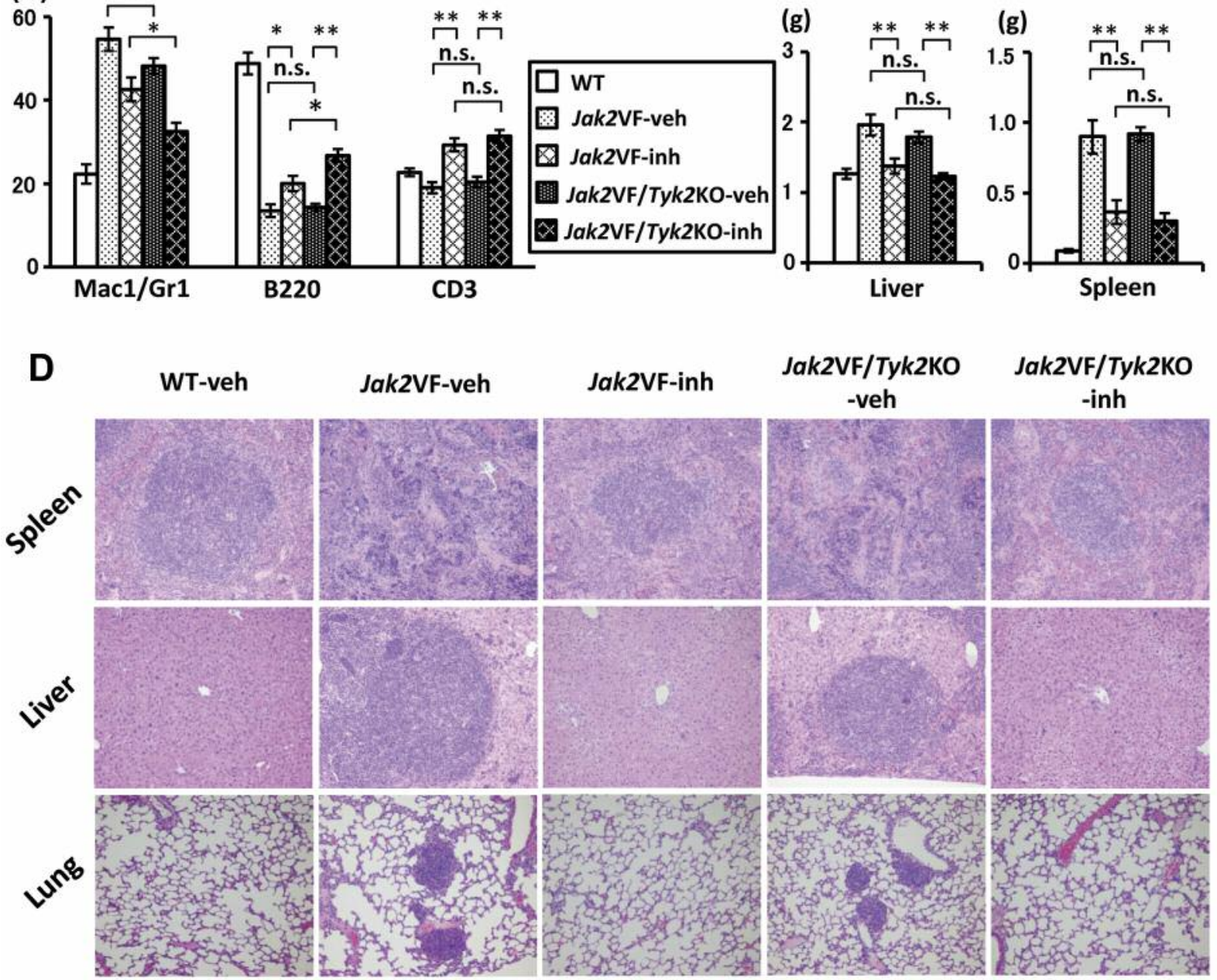
stages, but the platelet count declined with time. JAK2 inhibitor treatment resulted in sustained thrombocytosis (Jak2VF: $p<0.05 ;$ Jak2VF/Tyk2KO: $p=0.370$ ) (Figure 2A) compared to the vehicle-treated counterparts. No difference in platelet count was observed between inhibitor-treated $J a k 2 \mathrm{VF}$ and Jak2 VF/Tyk2KO mice.

The spleen weight was markedly increased by 10.4 -fold in vehicle-treated Jak2VF mice and 10.2-fold in $J a k 2 \mathrm{VF} / T y k 2 \mathrm{KO}$ vehicle-treated mice compared to vehicletreated WT mice. A significant reduction of spleen weight by 4.1 -fold in inhibitor-treated Jak2VF mice $(p<0.01)$ and by 3.4-fold in Jak2VF/Tyk2KO inhibitor-treated mice $(p<0.01)$ compared to vehicle-treated WT mice. There was no difference in improvement of splenomegaly between Jak2 VF and Jak2 VF/Tyk2KO mice upon administration of JAK2 inhibitor (Figure 2C), and there was also no difference in liver weight between those groups of mice (Figure 2C). Because the observation period was short, no difference was observed among groups regarding survival (data not shown).

Histological examination showed that myeloid cells markedly infiltrated the spleen, and the follicular structure had disappeared in vehicle-treated Jak2 VF and Jak2 VF/Tyk2KO mice (Figure 2D), but JAK2 inhibitor therapy improved cell invasion and restored follicular structure in both groups of mice (Figure 2D). In the liver and lung, cell infiltration was observed in the vehicle-treated groups (Figure 2D), but cell infiltration completely disappeared in the JAK2 inhibitortreated groups (Figure 2D). In both inhibitor-treated Jak2VF and Jak2 VF/Tyk2KO mice, histological improvement upon JAK2 inhibitor therapy was observed equally. In contrast to the drastic pathological improvement of these organs, JAK2 inhibitor had little effect on fibrosis in the BM, and TYK2 loss did not affect these result (data not shown). Constitution of hematopoietic cells in the BM and spleen was examined by FACS analysis (Table I). The number of BM cells in vehicletreated Jak2 VF and Jak2 VF/Tyk2KO mice decreased compared with the corresponding WT mice due to fibrotic changes, and consistent with pathological findings, cellularity

\section{$\leftarrow$}

Figure 2. Loss of tyrosine kinase 2 (TYK2) does not affect the therapeutic efficacy of a Janus kinase 2 (JAK2) inhibitor. A: Differential blood cell count every 4 weeks after drug administration ( $n=16$ in each group). ANOVA with repeated measures was used. B: Hematopoietic compartment of peripheral blood assessed by flow cytometry 16 weeks after drug administration ( $n=10$ in each group). Tukey's HSD test with one-way ANOVA was used. C: Spleen and liver weights 16 weeks after drug administration ( $n=10$ in each group). Tukey's HSD test with oneway ANOVA was used. D: Histological analysis 16 weeks after drug administration. WBCs, White blood cells; Hb, hemoglobin; Plt, platelets. Data are presented as the mean \pm SEM. Significantly different at $* p<0.05$ and $* * p<0.01$. did not recover with JAK2 inhibitor therapy. The number of cells in the spleen of vehicle-treated Jak2VF and $J a k 2 \mathrm{VF} / T y k 2 \mathrm{KO}$ mice increased because of extramedullary hematopoiesis, which was reduced with JAK2 inhibitor, although not with statistical significance. TYK2 loss did not affect cellularity in the BM or spleen. In Jak2VF and Jak2 VF/Tyk2KO mice, JAK2 inhibitor therapy significantly increased common myeloid progenitors in the BM and significantly reduced cluster of differentiation 71positive/lymphocyte antigen 76 (Ly76, also known as Ter119) -positive $\left(\mathrm{CD} 71^{+} / \mathrm{Ter} 119^{+}\right)$erythroid progenitors in the BM and spleen, although the biological significance of this is unknown. Both inhibitor-treated Jak2 VF and Jak2 VF/Tyk2KO mice showed similar changes upon JAK2 inhibitor administration compared with vehicle-treated groups, and there was no significant difference observed between inhibitor-treated Jak2VF and Jak2VF/Tyk2KO mice (Table I).

\section{Discussion}

In this study, we demonstrated the role of TYK2 in Jak2VFinduced MPN in vivo. In addition, the disease severity and treatment effect by JAK2 inhibitor were found to be comparable between Jak2 VF and Jak2 VF/Tyk2KO mice. Thus, our results indicate that TYK2 has no cooperative effects with JAK2VF at the onset of MPN nor upon treatment with a JAK2 inhibitor.

JAK2 inhibitors have shown excellent therapeutic effects on MPN symptoms in clinical trials $(2,3)$, but the plateauing of effects observed in these trials strongly indicates the presence of resistance to the inhibitor. Such a plateau of effects with regard to leukocyte count and organomegaly was also observed in our mouse models (5). Koppikar et al. revealed a mechanism of resistance in vitro using MPN cell lines and cells from patients treated with ruxolitinib. In the presence of JAK2 inhibitors, JAK2 does not have kinase activity itself, but rather, it forms a heterodimer with TYK2 or JAK1 and can enhance downstream signaling (4). In a clinical trial with momelotinib, which showed a strong inhibition profile of JAK1/JAK2/TYK2, a plateau effect on organomegaly and on the allele burden was also observed (9), suggesting that it is not the suppression of kinase activity but rather the reduction of protein expression that is necessary for the reversal of resistance. JaklKO mice showed perinatal lethality and defect in lymphoid development (10). On the other hand, Tyk2KO mice survived for a long time with no immunodeficiency (6). TYK2 is a good target of treatment, as its inhibition leads to fewer safety concerns than JAK1 inhibition Thus, it would be an ideal target for MPN treatment because long-term therapy is likely to be required, as in the case of imatinib mesylate treatment for $B C R-A B L-$ positive chronic myeloid leukemia $(11,12)$. Koppikar et al. 
Table I. Frequency of hematopoietic cells in mice treated with vehicle or Janus kinase 2 (JAK2) inhibitor.

\begin{tabular}{|c|c|c|c|c|c|c|c|c|c|c|}
\hline & \multicolumn{5}{|c|}{ Bone marrow } & \multicolumn{5}{|c|}{ Spleen } \\
\hline & \multirow{2}{*}{$\frac{\text { WT }}{\text { Vehicle }}$} & \multicolumn{2}{|c|}{$J a k 2 \mathrm{VF}$} & \multicolumn{2}{|c|}{$J a k 2 \mathrm{VF} / T y k 2 \mathrm{KO}$} & \multirow{2}{*}{$\begin{array}{c}\text { WT } \\
\text { Vehicle }\end{array}$} & \multicolumn{2}{|c|}{$J a k 2 \mathrm{VF}$} & \multicolumn{2}{|c|}{$J a k 2 \mathrm{VF} / T y k 2 \mathrm{KO}$} \\
\hline & & Vehicle & Inhibitor & Vehicle & Inhibitor & & Vehicle & Inhibitor & Vehicle & Inhibitor \\
\hline $\begin{array}{l}\text { Total cellularity } \\
\left(\times 10^{6}\right)\end{array}$ & $20.7 \pm 2.1$ & $8.1 \pm 0.96^{\dagger}$ & $6.3 \pm 0.43^{\dagger}$ & $6.8 \pm 0.45^{\dagger}$ & $6.6 \pm 0.65^{\dagger}$ & $6.0 \pm 0.29$ & $11.8 \pm 1.1^{\dagger}$ & $9.7 \pm 1.2^{\dagger}$ & $13.6 \pm 1.5^{\dagger}$ & $11.2 \pm 1.3^{\dagger}$ \\
\hline LT-HSC & $0.77 \pm 0.01$ & $0.02 \pm 0.01^{\dagger}$ & $0.03 \pm 0.01^{\dagger}$ & $0.03 \pm 0.01^{\dagger}$ & $0.04 \pm 0.01^{\dagger}$ & $0.01 \pm 0.001$ & $0.01 \pm 0.002$ & $0.01 \pm 0.004$ & $0.01 \pm 0.003$ & $0.01 \pm 0.002$ \\
\hline ST-HSC & $0.14 \pm 0.02$ & $0.02 \pm 0.003$ & $0.03 \pm 0.01$ & $0.02 \pm 0.01$ & $0.07 \pm 0.02$ & $0.01 \pm 0.01$ & $0.07 \pm 0.02^{\dagger}$ & $0.03 \pm 0.01$ & $0.04 \pm 0.01$ & $0.02 \pm 0.005$ \\
\hline MPP & $0.47 \pm 0.04$ & $0.48 \pm 0.08$ & $0.57 \pm 0.06$ & $0.38 \pm 0.05$ & $0.49 \pm 0.10$ & $0.30 \pm 0.02$ & $0.55 \pm 0.07 \dagger$ & $0.56 \pm 0.06^{\dagger}$ & $0.44 \pm 0.04$ & $0.52 \pm 0.06^{\dagger}$ \\
\hline CMP & $0.53 \pm 0.04$ & $0.29 \pm 0.04$ & $0.66 \pm 0.06^{*}$ & $0.48 \pm 0.08$ & $0.70 \pm 0.09 *$ & $0.02 \pm 0.002$ & $0.14 \pm 0.05^{\dagger}$ & $0.11 \pm 0.02^{\dagger}$ & $0.11 \pm 0.02^{\dagger}$ & $0.13 \pm 0.02 \dagger$ \\
\hline GMP & $2.5 \pm 0.14$ & $3.0 \pm 0.34$ & $3.3 \pm 0.15$ & $3.5 \pm 0.33$ & $3.2 \pm 0.34$ & $0.06 \pm 0.005$ & $0.31 \pm 0.09^{\dagger}$ & $0.15 \pm 0.02$ & $0.28 \pm 0.07^{\dagger}$ & $0.22 \pm 0.04$ \\
\hline MEP & $0.90 \pm 0.03$ & $0.91 \pm 0.16$ & $0.71 \pm 0.07$ & $0.58 \pm 0.05$ & $0.73 \pm 0.11$ & $0.28 \pm 0.02$ & $1.1 \pm 0.28^{\dagger}$ & $0.88 \pm 0.11^{\dagger}$ & $0.78 \pm 0.10$ & $0.72 \pm 0.06^{\dagger}$ \\
\hline MKP & $0.14 \pm 0.02$ & $0.06 \pm 0.02^{\dagger}$ & $0.07 \pm 0.01^{\dagger}$ & $0.05 \pm 0.005^{\dagger}$ & $0.10 \pm 0.02 *$ & $0.01 \pm 0.001$ & $0.66 \pm 0.02^{\dagger}$ & $0.11 \pm 0.02^{\dagger}$ & $0.04 \pm 0.01$ & $0.13 \pm 0.02^{\dagger *}$ \\
\hline CLP & $0.19 \pm 0.03$ & $0.05 \pm 0.01^{\dagger}$ & $0.07 \pm 0.01^{\dagger}$ & $0.05 \pm 0.01^{\dagger}$ & $0.07 \pm 0.01^{\dagger}$ & $0.01 \pm 0.002$ & $0.04 \pm 0.02$ & $0.01 \pm 0.003$ & $0.01 \pm 0.002$ & $0.02 \pm 0.01$ \\
\hline Mac1+Gr1+ & $67.2 \pm 0.93$ & $79.8 \pm 2.6^{\dagger}$ & $85.4 \pm 0.53^{\dagger}$ & $82.9 \pm 2.3^{\dagger}$ & $83.2 \pm 1.6^{\dagger}$ & $5.4 \pm 0.66$ & $19.0 \pm 4.3^{\dagger}$ & $14.0 \pm 1.8^{\dagger}$ & $16.6 \pm 1.8^{\dagger}$ & $14.7 \pm 2.2^{\dagger}$ \\
\hline B220+ & $16.5 \pm 1.2$ & $4.7 \pm 0.81^{\dagger}$ & $5.1 \pm 0.58^{\dagger}$ & $3.8 \pm 0.51^{\dagger}$ & $5.7 \pm 0.48^{\dagger}$ & $58.3 \pm 1.2$ & $39.9 \pm 3.2 \dagger$ & $45.5 \pm 1.4^{\dagger}$ & $44.3 \pm 3.1^{\dagger}$ & $46.0 \pm 1.1^{\dagger}$ \\
\hline $\mathrm{CD} 3+$ & $4.2 \pm 0.25$ & $3.3 \pm 0.72$ & $3.0 \pm 0.41$ & $2.5 \pm 0.41$ & $3.7 \pm 0.48$ & $26.9 \pm 1.1$ & $27.6 \pm 2.0$ & $33.8 \pm 2.3$ & $26.9 \pm 1.6$ & $33.8 \pm 2.7 *$ \\
\hline CD71+TER119+ & $30.9 \pm 0.96$ & $36.3 \pm 2.7$ & $25.0 \pm 2.3^{*}$ & $29.6 \pm 2.8$ & $21.5 \pm 1.7^{\dagger *}$ & $25.3 \pm 1.1$ & $29.5 \pm 2.9$ & $17.6 \pm 1.2^{\dagger *}$ & $24.2 \pm 2.2$ & $17.4 \pm 1.6^{*}$ \\
\hline CD41+ & $1.3 \pm 0.12$ & $5.8 \pm 1.3^{\dagger}$ & $7.6 \pm 1.1^{\dagger}$ & $5.8 \pm 0.79^{\dagger}$ & $8.1 \pm 1.8^{\dagger}$ & $1.4 \pm 0.13$ & $13.2 \pm 2.0^{\dagger}$ & $13.6 \pm 1.9^{\dagger}$ & $10.7 \pm 1.9^{\dagger}$ & $14.2 \pm 1.6^{\dagger}$ \\
\hline
\end{tabular}

Jak2VF: Janus kinase 2 V617F transgenic; Tyk2KO: tyrosine kinase 2 gene knockout; LT-HSC: long-term hematopoietic stem cells, $\mathrm{Lin}^{-} \mathrm{Sca}{ }^{+}{ }^{+} \mathrm{Kit}^{+} \mathrm{CD} 48^{-} \mathrm{CD} 150^{+}$; ST-HSC: short-term hematopoietic stem cells, Lin ${ }^{-} \mathrm{Sca}{ }^{+}{ }^{+} \mathrm{Kit}^{+} \mathrm{CD} 48^{-} \mathrm{CD} 150^{-}$; MPP: multipotent progenitor, $\mathrm{Lin}^{-} \mathrm{Sca}{ }^{+}{ }_{\mathrm{cKit}}{ }^{+} \mathrm{CD} 48^{+} \mathrm{CD} 150^{-}$; CMP: common myeloid progenitor, Lin ${ }^{-} \mathrm{Sca} 1^{-} \mathrm{cKit}^{+} \mathrm{CD} 34{ }^{+} \mathrm{Fc} \gamma \mathrm{R}^{\mathrm{lo}}$; GMP: granulocyte-macrophage progenitor, $\mathrm{Lin}^{-} \mathrm{Sca}{ }^{-}{ }^{-} \mathrm{Kit}^{+} \mathrm{CD} 34^{+} \mathrm{Fc} \gamma \mathrm{R} \mathrm{R}^{\mathrm{hi}}$; MEP: megakaryocyte-erythroid progenitor, $\mathrm{Lin}^{-} \mathrm{Sca}{ }^{-}{ }^{-} \mathrm{Kit}^{+} \mathrm{CD} 34-\mathrm{Fc} \gamma \mathrm{R}^{-/ l o}$. Data are the mean frequency $(\%) \pm \mathrm{SEM}$. The statistical significance of differences was assessed by non-repeated measures ANOVA and Tukey's HSD test: $\dagger^{\dagger}<0.05 v s$. vehicle-treated WT, ${ }^{*} p<0.05$ vehicle-treated $v s$. corresponding inhibitor-treated cells.

also showed that the knockdown of JAK1 or JAK1 and TYK2 by siRNA resensitized cells that had acquired resistance to ruxolitinib, whereas the knockdown of only TYK2 did not resensitize the cells (4). Our in vivo results are consistent with this study. TYK2 protein may play an auxiliary role in resistance to JAK2 inhibitor, and JAK1 protein might be the main player. Taken together, in combination with JAK2 inhibitor therapy, the results of this study suggest that TYK2 protein is not a sufficient therapeutic target alone; however, novel treatment strategies targeting JAK1 or JAK1 and TYK2 protein may be promising, although there is a risk of immunodeficiency.

\section{Conflicts of Interest}

The Authors declare no competing financial interests in regard to this study.

\section{Acknowledgements}

The Authors thank M. Matsushita, T. Shinmori, and S. Saito for their technical assistance. This work was supported by Grants-inAid for Young Scientists (B) $(26860738,15 \mathrm{~K} 19554)$ and for Scientific Research (C) (26461408) from the Japan Society for the Promotion of Science, and by Research Grants from Takeda Science Foundation.

\section{References}

1 Vainchenker W, Delhommeau F, Constantinescu SN and Bernard OA: New mutations and pathogenesis of myeloproliferative neoplasms. Blood 118: 1723-1735, 2011.

2 Verstovsek S, Mesa RA, Gotlib J, Levy RS, Gupta V, DiPersio JF, Catalano JV, Deininger M, Miller C, Silver RT, Talpaz M, Winton EF, Harvey JH, Arcasoy MO, Hexner E, Lyons RM, Paquette R, Raza A, Vaddi K, Erickson-Viitanen S, Koumenis IL, Sun W, Sandor V and Kantarjian HM: A double-blind, placebo-controlled trial of ruxolitinib for myelofibrosis. New Eng J Med 366: 799-807, 2012.

3 Harrison C, Kiladjian JJ, Al-Ali HK, Gisslinger H, Waltzman R, Stalbovskaya V, McQuitty M, Hunter DS, Levy R, Knoops L, Cervantes F, Vannucchi AM, Barbui T and Barosi G: JAK inhibition with ruxolitinib versus best available therapy for myelofibrosis. N Engl J Med 366: 787-798, 2012.

4 Koppikar P, Bhagwat N, Kilpivaara O, Manshouri T, Adli M, Hricik T, Liu F, Saunders LM, Mullally A, Abdel-Wahab O, Leung L, Weinstein A, Marubayashi S, Goel A, Gonen M, Estrov Z, Ebert BL, Chiosis G, Nimer SD, Bernstein BE, Verstovsek $S$ and Levine RL: Heterodimeric JAK-STAT activation as a mechanism of persistence to JAK2 inhibitor therapy. Nature 489: 155-159, 2012.

5 Shide K, Kameda T, Markovtsov V, Shimoda HK, Tonkin E, Fang S, Liu C, Gelman M, Lang W, Romero J, McLaughlin J, Bhamidipati S, Clough J, Low C, Reitsma A, Siu S, Pine P, Park G, Torneros A, Duan M, Singh R, Payan DG, Matsunaga T, 
Hitoshi Y and Shimoda K: R723, a selective JAK2 inhibitor, effectively treats Jak2V617F-induced murine myeloproliferative neoplasm. Blood 117: 6866-6875, 2011.

6 Shimoda K, Kato K, Aoki K, Matsuda T, Miyamoto A, Shibamori M, Yamashita M, Numata A, Takase K, Kobayashi S, Shibata S, Asano Y, Gondo H, Sekiguchi K, Nakayama K, Nakayama T, Okamura T, Okamura S, Niho Y and Nakayama K: Tyk2 plays a restricted role in IFN alpha signaling, although it is required for IL-12-mediated T-cell function. Immunity 13: 561-571, 2000.

7 Nakaya Y, Shide K, Niwa T, Homan J, Sugahara S, Horio T, Kuramoto K, Kotera T, Shibayama H, Hori K, Naito H and Shimoda K: Efficacy of NS-018, a potent and selective JAK2/Src inhibitor, in primary cells and mouse models of myeloproliferative neoplasms. Blood Cancer J 1: e29, 2011.

8 Shide K, Kameda T, Yamaji T, Sekine M, Inada N, Kamiunten A, Akizuki K, Nakamura K, Hidaka T, Kubuki Y, Shimoda H, Kitanaka A, Honda A, Sawaguchi A, Abe H, Miike T, Iwakiri H, Tahara Y, Sueta M, Hasuike S, Yamamoto S, Nagata K and Shimoda K: Calreticulin mutant mice develop essential thrombocythemia that is ameliorated by the JAK inhibitor ruxolitinib. Leukemia 31(5): 1136-1144, 2017.

9 Gupta V, Mesa RA, Deininger MWN, Rivera CE, Sirhan S, Brachmann CB, Collins H, Kawashima J, Xin Y and Verstovsek $\mathrm{S}$ : A phase $1 / 2$, open-label study evaluating twice-daily administration of momelotinib in myelofibrosis. Haematologica 102: 94, 2016.
10 Rodig SJ, Meraz MA, White JM, Lampe PA, Riley JK, Arthur CD, King KL, Sheehan KC, Yin L, Pennica D, Johnson EM, Jr. and Schreiber RD: Disruption of the Jak1 gene demonstrates obligatory and nonredundant roles of the Jaks in cytokineinduced biologic responses. Cell 93: 373-383, 1998.

11 Pinilla-Ibarz J, Sweet K, Emole J and Fradley M: Long-term BCR-ABL1 tyrosine kinase inhibitor therapy in chronic myeloid leukemia. Anticancer Res 35: 6355-6364, 2015.

12 Iriyama N, Hatta Y, Kobayashi S, Uchino Y, Miura K, Kurita D, Kodaira H, Takahashi H, Iizuka Y, Inoue M and Takei M: Higher red blood cell distribution width is an adverse prognostic factor in chronic-phase chronic myeloid leukemia patients treated with tyrosine kinase inhibitors. Anticancer Res 35: 5473-5478, 2015.
Received May 2, 2017

Revised May 25, 2017

Accepted May 30, 2017 